\title{
COMPACTACIÓN INDUCIDA POR EL TRÁNSITO VEHICULAR SOBRE UN SUELO EN PRODUCCIÓN HORTÍCOLA
}

\author{
Antonino Marcelo Terminiello ${ }^{1}$, Roberto Hernan. Balbuena ${ }^{1}$, Jorge Alejandro Claverie $^{1}$ \& \\ Juan Pablo Casado
}

\begin{abstract}
RESUMEN
Se realizaron ensayos en campo con el objeto de caracterizar el estado de compactación de un suelo en producción hortícola inducida por el tránsito, luego del desarrollo del ciclo de un cultivo de repollo (Brassica oleracea L. grupo capitata ) y su incidencia sobre el rendimiento. Se efectuaron determinaciones de resistencia a la penetración, densidad aparente y humedad gravimétrica sobre el suelo y biomasa aérea al finalizar el ciclo del cultivo en los sectores de mayor y menor número de pasajes de vehículos. Los valores de resistencia a la penetración fueron de 1,7 y 1,3 $\mathrm{MPa}$, significativamente mayores para el tratamiento de más de 7 y 3 pasadas respectivamente en el rango de profundidad de $0-100 \mathrm{~mm}$. No se manifestaron diferencias en el parámetro densidad aparente en la totalidad del perfil. Se encontraron diferencias estadísticamente significativas en biomasa aérea, para el tratamiento de 3 pasadas (1902.6 $\left.\mathrm{g} \mathrm{planta}^{-1}\right)$, en relación al de mas de 7 pasadas $\left(1447,9 \mathrm{~g} \mathrm{planta}^{-1}\right)$. El pasaje repetido sobre los surcos originan incrementos en la resistencia a la penetración a nivel superficial. El peso fresco y la materia seca del cultivo son afectados por el número de pasadas de los tractores y máquinas agrícolas.
\end{abstract}

Palabras clave: compactación de suelo, resistencia a la penetración, biomasa aérea, repollo

\section{INDUCED COMPACTION BY THE VEHICULAR TRAFFIC IN A SOIL FOR HORTICULTURAL CROP PRODUCTION}

\begin{abstract}
Field tests were carried out with the objective of characterizing the state of compaction in horticultural soil induced by traffic, after the development of a cabbage crop (Brassica oleracea L. group capitata) cycle, and its incidence on yield. Measurements of penetration resistance, bulk density, and moisture content in soil were made and aeread biomass at the end of the cycle crop in sectors with a larger and smaller number of vehicles passes. Values of penetration resistance of 1.7 and 1.3 MPa were found for treatment with more than 7 passes and 3 passes, respectively, in the depth range of $0-100 \mathrm{~mm}$. No significant differences were found in bulk density parameter in the entire soil profile. Statistically, significant differences were found for aereal biomass values of 3 passes treatment $\left(1902.6 \mathrm{~g} \mathrm{plant}^{-1}\right)$, in relation to more than 7 passes $\left(1447.9 \mathrm{~g} \mathrm{plant}^{-1}\right)$. Repeated passes on rows resulted in increments of penetration resistance at shallow depths. The number of the tractor and agricultural machinery passes influenced the fresh and dry weight of crop.
\end{abstract}

Key words: soil compaction, penetration resistance, biomass, cabbage

Recebido em 07/12/1999, Protocolo 140/99

${ }^{1}$ Docentes-investigadores de la Facultad de Cs Agrarias y Forestales de la Universidad Nacional de La Plata. Avda 60 y 119 (1900) La Plata, Argentina. Fax: (54 221) 425 2346.E-mail: amtermi@ceres.agro.unlp.edu.ar, balbuena@ceres.agro.unlp.edu.ar, jotaclaave@usa.netejpcasado@yahoo.com 


\section{INTRODUCCIÓN}

La compactación del suelo en condiciones de campo es comunmente causada por la labranza y el tráfico de ruedas (Voorhees,1989), pudiendo reducir substancialmente el rendimiento de los cultivos. La compactación disminuye el volumen de poros totales y produce cambios en la distribución del tamaño de los mismos, ocasionando un mayor porcentaje de poros pequeños (Breland \& Hansen, 1996).

Para un suelo dado, la distribución de la compactación en el perfil es función de la carga aplicada, la presión ejercida por el rodado, el estado de humedad y la intensidad de tránsito recibida (Raper \& Erbach, 1990). Smith \& Dickson (1990) informaron que la compactación superficial se produce por la presión en la zona de contacto rueda-suelo, pero la subsuperficial es producida por el peso total del equipo.

Voorhees (1989) determinó que vehículos con peso por eje que no exceden los $5 \mathrm{Mg}$ producirían compactación solamente en los primeros $30 \mathrm{~cm}$ del perfil del suelo.

Los parámetros usualmente utilizados para la medición de los efectos del tráfico con altas cargas sobre las propiedades del suelo son la densidad aparente y la resistencia a la penetración (Alakukku, 1997); de los mismos, el primero ha sido indicado como menos sensible para la determinación de la compactación inducida por tránsito (Alakukku, 1996). Threadgill (1982) indicó que valores de índice de cono de 1,5 MPa pueden reducir el crecimiento de las raíces, mientras que valores superiores a 2,1 MPa lo limitarían. La compactación del suelo restringiría la distribución de raíces en el perfil del suelo (Hakansson et al., 1988), por lo cual se afectaría, en consecuencia, la eficiencia de extracción desde el suelo de nutrientes y agua por las plantas, puesto que aquella depende de la concentración y tamaño de la rizósfera según Smitle \& Williamson (1977).

La respuesta de los cultivos a la compactación inducida por tránsito ha sido ampliamente estudiada (Brereton et al., 1986; Hakansson et al. 1988; Henderson, 1991). El efecto es sumamente variable en función de la especie en particular y de las condiciones del medio ambiente. Orzolek (1991), comparando la respuesta a la compactación del rendimiento de distintos cultivos hortícolas, encontró reducciones del $65 \%$ para repollo, $65 \%$ para pepino y $55 \%$ para maíz dulce. Wolfe et al.(1995), en ensayos de tres años, cuantificó reducciones en biomasa del tratamiento compactado del orden del $63 \%$ para repollo de siembra directa y del $29 \%$ para repollo de transplante.

Stone (1988) determinó un decrecimiento en el rendimiento de cultivos de habas (Vicia faba), puerros (Allium porrum), repollos (Brassica oleracea var capitata) y remolachas (Beta vulgaris var esculenta) para los tratamientos compactados. El mismo autor cita una reducción de materia seca de alrededor de 1 tha ${ }^{-1}$ por $0,5 \mathrm{MPa}$ de incremento de resistencia a la penetración en el intervalo de 0,5 a 2,5 MPa., no encontrando la misma relación con el parámetro densidad aparente. La producción de materia seca de los cuatro cultivos fue escasamente afectada por valores de resistencia a la penetración entre 1 y $1,5 \mathrm{MPa}$, pero notablemente reducida con resistencias mayores.

La mayoría de las los trabajos que estudian el efecto de la compactación en los cultivos lo hacen sobre condiciones de compactación simuladas y no sobre sistemas reales de producción. En virtud de lo anteriormente señalado, el objetivo de este trabajo es la caracterización del estado de compactación de un suelo hortícola, debido al tránsito de las labores, luego del desarrollo de un ciclo de cultivo.

\section{MATERIAL Y MÉTODOS}

El ensayo fue conducido en un establecimiento hortícola ubicado en el paraje «El Peligro» perteneciente al partido de La Plata, provincia de Buenos Aires, República Argentina .

El experimento se realizó sobre un suelo de clase textural franco arcilloso con $24 \%$ de arena, $47 \%$ de limo y $29 \%$ de arcilla en una parcela de producción con un ancho de $19 \mathrm{~m}$ y una longitud de $80 \mathrm{~m}$. Sobre la misma se estableció un cultivo de repollo (Brassica olerácea L. grupo capitata), de transplante, con un marco de plantación de $750 \mathrm{~mm}$ entre hileras y $400 \mathrm{~mm}$ entre plantas.

Las características de los tractores utilizados (Tabla 1) y las labores realizadas fueron las siguientes:

Del seguimiento de las labores sobre la parcela se identificaron lugares del terreno con diferentes intensidades de tránsito a partir del momento de finalización de la preparación del terreno para la realización de los surcos, tomándose como tratamientos a: 1) el sector central del camellón donde se ubican las plantas (lomo), 2) los surcos que tuvieron durante el ciclo del cultivo 3 pasadas (mínimo número de pasajes) de conjuntos tractor implemento y 3 ) surcos por donde se efectuaron más de 7 pasadas (máximo número de pasajes).

Para el análisis de las modificaciones generadas sobre el suelo por el tránsito se midieron las siguientes variables experimentales:

Resistencia a la penetración con penetrómetro de cono bajo norma ASAE S 313,2 (ASAE, 1992), cada $25 \mathrm{~mm}$ hasta una profundidad de $600 \mathrm{~mm}$.

Densidad aparente con sonda núcleo gammamétrica TROXLER 3440, cada $50 \mathrm{~mm}$ hasta $300 \mathrm{~mm}$ de profundidad.

Humedad del suelo, por el método de gravimétrico a intervalos de $100 \mathrm{~mm}$ de hasta los $500 \mathrm{~mm}$.

Tabla 1. Descripción de los tractores y equipos que intervinieron durante el ciclo del cultivo

\begin{tabular}{|c|c|c|c|c|c|c|}
\hline Tractor & $\begin{array}{c}\text { Peso eje } \\
\text { Delantero } \\
(\mathrm{kN})\end{array}$ & $\begin{array}{c}\text { Peso eje } \\
\text { Trasero } \\
(\mathrm{kN})\end{array}$ & $\begin{array}{c}\text { Rodado } \\
\text { Delantero }\end{array}$ & $\begin{array}{l}\text { Rodado } \\
\text { Trasero }\end{array}$ & $\begin{array}{l}\text { Trocha } \\
(\mathrm{mm})\end{array}$ & $\begin{array}{c}\text { Labor } \\
\text { Realizada } \\
\left(\mathrm{n}^{\circ} \text { de eventos }\right)\end{array}$ \\
\hline Fiat $450 \mathrm{R}$ & 7 & 13,5 & $600-16$ & $13,6-18$ & 1520 & $\begin{array}{c}\text { Tratamientos } \\
\text { fitosanitarios (4) }\end{array}$ \\
\hline Fiat $400 \mathrm{E}$ & 8,4 & 11,5 & $6,50-16$ & $9,5-36$ & 1500 & Escardillada (4) \\
\hline Fiat 540 & 7,6 & 12,9 & $600-16$ & $14,9-26$ & 1540 & Surqueado (1) \\
\hline Zanello V210 & 9,4 & 26,6 & $750-16$ & $18,4-34$ & 1830 & $\begin{array}{c}\text { Disqueado (4) } \\
\text { Vibrocultivador (2) }\end{array}$ \\
\hline M. Ferguson 265 & 8 & 21,5 & $6,5-16$ & $18,4-30$ & 1570 & Escarificador (2) \\
\hline
\end{tabular}


Sobre los lomos adyacentes a los tratamientos con tránsito se recolectaron al azar plantas (10 repeticiones para cada situación de tránsito), sobre las cuales se determinaron el peso fresco y el peso la materia seca de la parte aérea.

El análisis estadístico de los datos se efectuó mediante análisis de la varianza, cuantificándose las diferencias mediante el test de Tukey.

\section{RESULTADOS Y DISCUSIÓN}

Del análisis de los valores de la Tabla 2 puede observarse un incremento estadísticamente significativo de la resistencia a la penetración verificado en el primer estrato de profundidad (0-100 mm), en relación a la situación de mayor número de pasadas.

Tabla 2. Medias de resistencia a la penetración $(\mathrm{MPa})^{*}$, por tratamientos y profundidades

\begin{tabular}{lccccc}
\hline \multirow{2}{*}{ Tratamientos } & \multicolumn{5}{c}{ Resistencia a la Penetración } \\
\cline { 2 - 6 } & $0-100$ & $100-200$ & $200-300$ & $300-400$ & $400-500$ \\
\cline { 2 - 6 } & $1,07 \mathrm{a}$ & $1,52 \mathrm{a}$ & $1,19 \mathrm{a}$ & $1,12 \mathrm{a}$ & $1,45 \mathrm{a}$ \\
Lomo & $1,30 \mathrm{a}$ & $1,46 \mathrm{a}$ & $1,07 \mathrm{a}$ & $1,20 \mathrm{a}$ & $1,49 \mathrm{a}$ \\
3 Pasadas & $1,70 \mathrm{~b}$ & $1,42 \mathrm{a}$ & $1,07 \mathrm{a}$ & $1,23 \mathrm{a}$ & $1,56 \mathrm{a}$ \\
Más de 7 pasadas & \multicolumn{5}{c}{ " } \\
"Valores seguidos de igual letra consignan diferencias no significativas (P<0,05) de acuerdo al test de \\
Tukey
\end{tabular}

La localización del mayor incremento de resistencia a la penetración en dicho estrato pudo deberse al tránsito de los tractores que realizaron los distintos trabajos culturales, luego del establecimiento del cultivo. Debido al peso por eje y a la presión de contacto en la interfase rueda-suelo que poseían los mismos manifestarían su efecto superficialmente, concordando con lo citado por Raper \& Erbach (1990) y Smith \& Dickson (1990).

En el estrato subsiguiente de profundidad $(100-200 \mathrm{~mm}) \mathrm{se}$ manifestó una condición de suelo más consolidado y en forma homogénea para los tres tratamientos, posiblemente atribuible a la existencia de un piso de labor, la cual podría haber determinado una situación de soporte que posibilitara el incremento de la resistencia a la penetración para el tratamiento de más pasadas ( $58 \%$ de incremento con respecto al lomo para el primer intervalo de profundidad). Lo anteriormente expresado se debería a un efecto de acumulación de la resistencia a la penetración hacia la superficie, no alcanzando a ser significativa la diferencia de $111 \%$ de incremento para el tratamiento de menor número de pasadas. Cabe aclarar que en la preparación del terreno previo a la realización de los surcos fue la modal para el establecimiento y se conformó con 2 pasadas de escarificador y 4 de rastra de discos con una profundidad de trabajo similar para ambas herramientas. Los tractores que realizaron las labores anteriormente mencionadas fueron los de mayor peso, estando el mismo por debajo de los $49 \mathrm{kN}$ por eje citados por Voorhees (1989) como peso causante de la compactación por tránsito en los primeros $300 \mathrm{~mm}$ del perfil.

La Tabla 3 muestra los valores de humedad para tratamientos y profundidades, los mismos pueden considerarse como normales para un perfil hortícola sometido a riegos periódicos durante el ciclo del cultivo. Dada la homogeneidad de los mismos, las diferencias de resistencia a la penetración podrían atribuirse fundamentalmente al tránsito de las diferentes labores y no a discontinuidades de humedad del perfil, dada la ingerencia de la misma sobre las determinaciones de índice de cono.

Tabla 3. Valores de humedad gravimétrica $(\%)^{*}$, por tratamientos

\begin{tabular}{lccccc}
\hline \multirow{2}{*}{ Tratamientos } & \multicolumn{5}{c}{ Humedad Gravimétrica } \\
\cline { 2 - 6 } & $0-100$ & $100-200$ & $200-300$ & $300-400$ & $400-500$ \\
\hline Lomo & $21,9 \mathrm{a}$ & $27,5 \mathrm{a}$ & $29,1 \mathrm{a}$ & $32,6 \mathrm{a}$ & $31,4 \mathrm{a}$ \\
3 Pasadas & $25,4 \mathrm{a}$ & $26,9 \mathrm{a}$ & $32,2 \mathrm{a}$ & $31,3 \mathrm{a}$ & $36,4 \mathrm{a}$ \\
Más de 7 pasadas & $24,2 \mathrm{a}$ & $28,4 \mathrm{a}$ & $32,2 \mathrm{a}$ & $35,9 \mathrm{a}$ & $36,4 \mathrm{a}$ \\
\hline
\end{tabular}

Valores seguidos de igual letra consignan diferencias no significativas $(\mathrm{P}<0,05)$ de acuerdo al test de Tukey

En la Figura1 se presentan los valores de densidad aparente relevados por tratamientos y por estratos. Si bien se puede verificar un incremento de los mismos en función de la profundidad, las medias alcanzadas no resultan estadísticamente significativas poniendo de manifiesto la menor sensibilidad del parámetro mencionado en poder detectar cambios en el perfil motivados por pasajes repetidos sobre el terreno de conjuntos tractor-implementos durante el ciclo del cultivo. Lo anteriormente expuesto concuerda con lo citado por Alakukku (1996), al comparar las determinaciones de densidad aparente en relación a la resistencia a la penetración y su capacidad para detectar compactación por el tránsito.

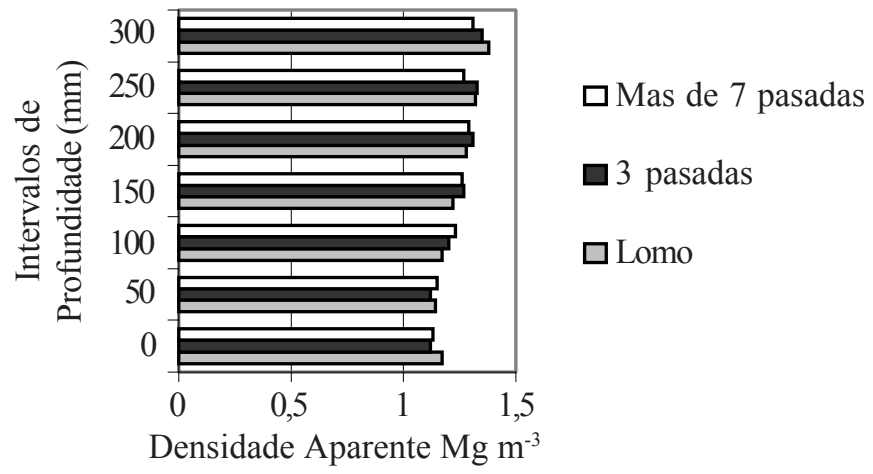

Figura 1. Valores de densidad aparente según tratamientos e intérvalos de profundidad

En la Tabla 4 puede apreciarse las diferencias estadísticamente significativas encontradas en la determinación de peso fresco y materia seca para los tratamientos, con diferencias porcentuales del $24 \%$ y del $20.2 \%$ respectivamente.

Tabla 4. Valores de biomasa aérea y materia seca de repollo para cada tratamiento de tránsito*

\begin{tabular}{lcc}
\hline \multirow{2}{*}{ Tratamientos } & Peso seco & Peso fresco \\
\cline { 2 - 3 } & \multicolumn{2}{c}{ g planta $^{-1}$} \\
\hline 3 Pasadas & $163,6 \mathrm{~b}$ & $1902,6 \mathrm{~b}$ \\
Más de 7 pasadas & $130,5 \mathrm{a}$ & $1447,9 \mathrm{a}$ \\
\hline $\begin{array}{l}\text { Valores seguidos de igual letra consignan diferencias no significativas (P }<0,05) \text { de acuerdo al test de } \\
\text { Tukey }\end{array}$
\end{tabular}

Las disminuciones en rendimiento fueron del 20,2 y $23,9 \%$ para peso seco y biomasa aérea respectivamente, dichos valores estarían dentro del rango de manifestado por Brereton et al. (1986); Hakansson et al. (1988); Henderson, (1991), encontrándose algo por debajo del $29 \%$ citado por Wolfe et al. (1995), al referirse a la reducción de rendimiento relevadas para el cultivo de repollo bajo la modalidad de transplante. 
Posiblemente la mayor resistencia a la penetración verificada en los surcos laterales a los lomos donde se recolectaron las plantas para la evaluación peso fresco y materia seca, hubo limitado el desarrollo radicular lateral y reducido consecuentemente la absorción de nutrientes y agua (Smitle \& Williamson (1977), Hakansson et al. (1988) motivando los valores expresados en la Tabla 4. Por otra parte, también pudo haberse reducido el desarrollo radicular en profundidad debido a la existencia de una capa entre los 100 y $200 \mathrm{~mm}$ de valores de resistencia a la penetración cercanos a 1,5 $\mathrm{MPa}$ citados por Threadgill (1982), como limitantes para el mismo. Las situaciones anteriormente manifestadas pudieron conjugarse y producir la disminución de la biomasa aérea.

Considerando rendimientos potenciales absolutos en peso fresco, se encontraron diferencias de aproximadamente $15 \mathrm{Mg} \mathrm{ha}^{-1}$ para un incremento de resistencia a la penetración de 0,4 MPa entre los tratamientos, para la densidad de plantación utilizada en el ensayo.

En relación a todo lo anteriormente mencionado, merecería tenerse en cuenta la posibilidad de considerar la implementación en sistemas de producción hortícolas de vías de tránsito controlado, readecuando anchos de trabajo y potencias de tractores para reducir el número de pasadas y minimizar las consecuencias de la compactación inducida por las labores culturales durante el desarrollo del cultivo.

\section{CONCLUSIONES}

1. En un sistema de explotación hortícola los mayores números de pasadas de ruedas sobre un surco originan incrementos en la resistencia a la penetración en superficie.

2. El pasaje repetido sobre los surcos del cultivo de repollo afecta el peso fresco y seco por planta.

3. La compactación inducida por el tránsito de vehículos se detecta con mayor claridad utilizando la evolución de la resistencia a la penetración que la densidad aparente.

\section{AGRADECIMIENTOS}

Los autores agradecen al Sr. Daniel Marozzi, propietario de la finca donde se realizó el trabajo, por la predisposición y buena voluntad manifestada en todo momento para los requerimientos del ensayo, posibilitando la realización del mismo en tiempo y forma.

\section{REFERENCIAS BIBLIOGRÁFICAS}

ALAKUKKU, L.. Persistence of soil compaction due to high axle load traffic. I Short-term effects on the properties of clay and organic soil. Soil and Tillage Research, Amsterdam, Netherlands. v.37, p.211-222. 1996
ALAKUKKU, L., Properties of fine-textured subsoils as affected by high axle load traffic. In: Academic Dissertation: Longterm soil compaction due to high axle load traffic. Helsinki, Finland: Agricultural Research Centre of Finland. Institute of Crop and Soil Science, 1997. p.61-71.

ASAE Standards. ASAE S 313 Soil cone penetrometer. St. Joseph, Michigan: ASAE, 1992.611p.

BRELAND T.A.; HANSEN S. Nitrogen mineralization and microbial biomass as affected by soil compaction. Soil Biolgy \& Biochemistry. Amsterdam, Netherlands. Ed. Elsevier. v.28, n.4, p.655-663. 1996.

BRERETON, J. C.; MCGOWAN M.; DAWKINS T.C.K.. The relative sensitivity of spring barley, spring field beans, and sugar beet crops to soil compaction. Field Crops Research, Amsterdam, Netherlands. v.13, p.223-232. 1986.

HAKANSSON, I.; VOORHEES W.B.; RILEY H.. Vehicle and wheel factors influencing soil compaction and crop response in different traffic regimes. Soil Tillage Research, Amsterdam, Netherlands. v.35.p.239-282. 1988.

HENDERSON, C.W.L. Sensitivity of eight cereal and legume species to the compaction status of deep, sandy soils. Australian Journal Experimental. Agriculture, Collingwood. v.31,p.347-355. 1991.

ORZOLEK, MD. Establishment of vegetables in the field. Hort Technology. Alexandria v.1, p.78-81. 1991.

RAPPER, R.; ERBACH, D. Prediction of soil stresses using the finite element method. Transaction of the ASAE. St. Joseph, v.33, n.3, p.725-730. 1990.

SMITH D. L.; DICKSON J.W. Contributions of weight and ground pressure to soil compaction. Journal of Agricultural Engineering Researchm, Silsoe, UK . v.43, p.13-29. 1990.

SMITTLE D.A.; WILLAMSON, R.E.. Effect of soil compaction on nitrogen and water use efficiency, root growth, yield, and fruit shape of pickling cucumbers. Journal of the American Society for Horticultural Science. Virginia, v.102, n.6, p.822-825. 1977.

STONE, D.A. Subsoil strength and yield of vegetable crops. Soil use and management, Oxford, London. v.4, n.1, p.10-14, 1988.

THREADGILL, E.D.. Residual tillage effects as determined by cone index. Transaction of the ASAE, St. Joseph, v.25, p.859-863. 1982.

UNGER, W.; KASPAR, C. Soil compaction and root growth: A Review. Agronomy Journal. Madison, Wisconsin. v. 86, p.759-766. 1994.

VOORHEES, W.B. Root activity related to shallow and deep compaction. In: Lasson, L.E. (ed.) Mechanics and related processes in structured agricultural soil. Morris, MN: Kleuwer Academic Publishers. 1989. p.173-186.

WOLFE, D, TOPOLESKID.T.; GUNDERSHEIMN. A.;. INGALL, B.A. Growth and yield sensitivity of four vegetable crops to soil compaction. Journal of American Society Horticultural Science, Virginia. v.120, n.6, p.956-963. 1995. 